Article

\title{
Untrimmed Low-Power Thermal Sensor for SoC in 22 nm Digital Fabrication Technology
}

\author{
Ro'ee Eitan and Ariel Cohen * \\ Intel Israel LTD., S.B.I. Park Har Hotzvim, CFF8, Jerusalem 91031, Israel; \\ E-Mail: roee.eitan@intel.com \\ * Author to whom correspondence should be addressed; E-Mail: ariel.cohen@intel.com; \\ Tel.: 972-2-589-7612; Mobile: 972-54-788-7612.
}

External Editor: Alexander Fish

Received: 30 September 2014; in revised form: 21 November 2014 / Accepted: 24 November 2014 / Published: 9 December 2014

\begin{abstract}
Thermal sensors (TS) are essential for achieving optimized performance and reliability in the era of nanoscale microprocessor and system on chip (SoC). Compiling with the low-power and small die area of the mobile computing, the presented TS supports a wide range of sampling frequencies with an optimized power envelope. The TS supports up to $45 \mathrm{~K}$ samples/s, low average power consumption, as low as $20 \mu \mathrm{W}$, and small core $\mathrm{Si}$ area of $0.013 \mathrm{~mm}^{2}$. Advanced circuit techniques are used in order to overcome process variability, ensuring inaccuracy lower than $\pm 2{ }^{\circ} \mathrm{C}$ without any calibration. All this makes the presented thermal sensor a cost-effective, low-power solution for $22 \mathrm{~nm}$ nanoscale digital process technology.
\end{abstract}

Keywords: thermal sensor; sigma-delta Analog to Digital Converter (ADC); low-power; untrimmed temperature sensor; nanoscale digital process

\section{Introduction}

Integrated thermal sensor (TS) circuits have become key elements in high performance systems, especially in processors and system on chip (SoC). Such applications require a relatively high 
precision thermal sensor (typically $\pm 3{ }^{\circ} \mathrm{C}$ in a wide temperature range), in order to achieve high reliability and performance [1,2]. The conventional method of thermal sensing is based on diode connected Bipolar Junction Transistor (BJT) voltage temperature dependence [2-7]. A base-emitter voltage equation of BJT transistor is (to first order approximation):

$$
V_{\mathrm{BE}}=\frac{n k T}{q} \operatorname{Ln}\left(\frac{I}{I_{0}}\right)
$$

where $n$ is the ideality factor of the diode, $k$ is Boltzmann's constant, $T$ is the absolute temperature, $q$ is the electron charge, $I_{0}$ is the diode's saturation current and $I$ is the current through the diode. This voltage is inversely proportional to the temperature, because of the strong temperature dependence of the saturation current $I_{0}$. The diode voltage is marked as $V_{\mathrm{BE}}$, since a diode connected BJT transistor is used in order to implement a diode, from this point onwards when referring to "diode" the meaning is diode connected BJT.

The differential voltage between the two diodes is:

$$
\Delta V_{\mathrm{BE}}=V_{\mathrm{BE} 2}-V_{\mathrm{BE} 1}=\frac{n k T}{q}\left[\ln \left(\frac{I_{2}}{I_{0}}\right)-\ln \left(\frac{I_{1}}{I_{0}}\right)\right]=\frac{n k T}{q} \ln (M)
$$

where $M$ is the ratio between the diodes' current density. This voltage is directly proportional to the temperature.

Several methods have previously been used to read out the diodes' voltage and convert it into a temperature reading. One of the popular implementations, used at industry, finds the crossing point between the amplified differential voltage of the two diodes, which is directly proportional to the temperature, and the voltage of a single diode, which is inversely proportional to the temperature (see Figure 1) [8]. For a given temperature, there will be exactly one crossing point, which exists only for a specific diodes' current. This current is controlled by a Digital to Analog Converter (DAC). A controller sweeps across the current DAC until this point is found by a comparator. The DAC code at this point represents the evaluated temperature [9]. This architecture is relatively limited in linearity and accuracy over a wide temperature range.

Moreover, there is a variance in the slope of the linear functions, due to sensitivity to process variations. The slope initial variation is large, requiring two-temperature calibration in order to achieve the required accuracy. Performing two temperature calibrations during High Volume Manufacturing (HVM) tests is considered costly.

Several motivations drove and guided the design of the temperature sensor presented in this paper: (a) to provide the best possible accuracy, as accurate temperature sensing extends performance of SoCs; (b) to utilize advanced circuit techniques to overcome process variance effects and, thus, to get a robust and accurate TS; (c) to reduce the products' HVM calibration process cycle to a single-temperature calibration or to no calibration; (d) to achieve goals (a) and (b) with the smallest possible power and area penalty; and (e) to be configurable in order to meet a wide range of power versus sampling rate optimized envelop for different requirements of several projects. 
Figure 1. Operation principle of thermal sensor $(\mathrm{CD}$ - current density; a-temperature coefficient of $\left.\Delta V_{\mathrm{BE}}\right)$.
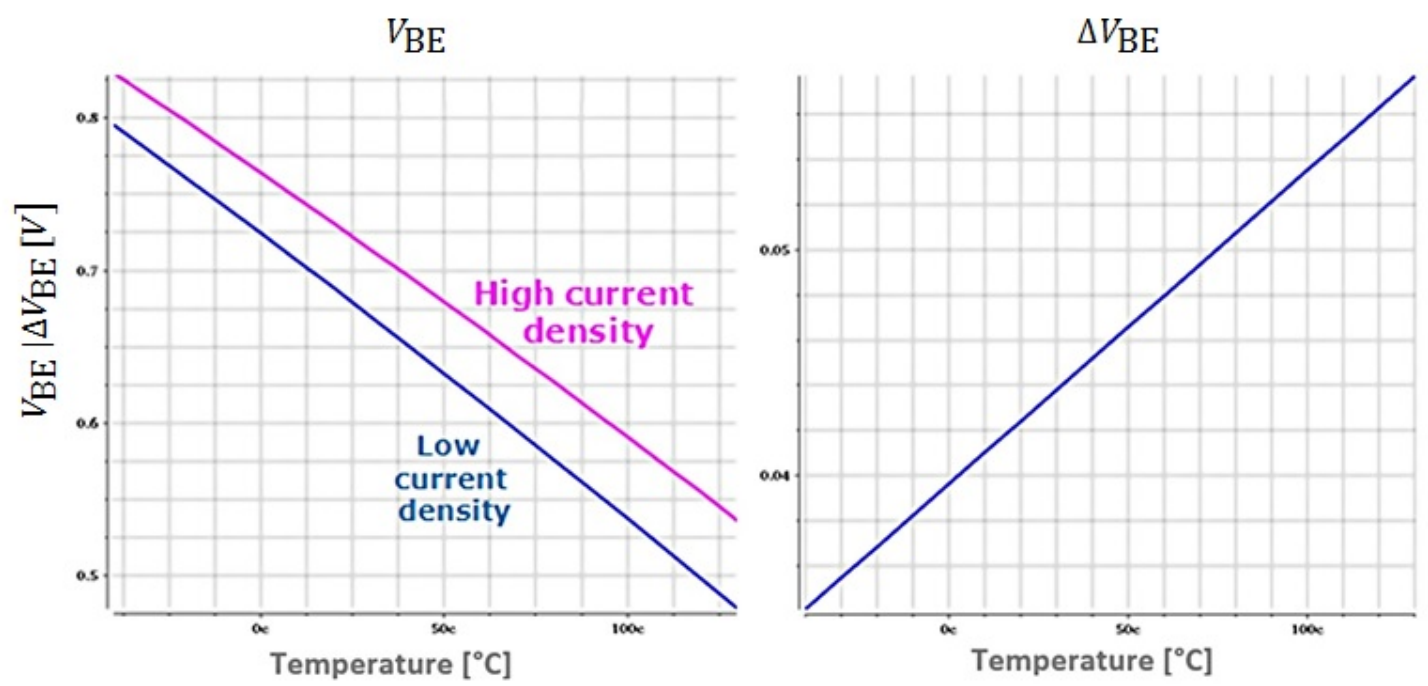

$$
\begin{array}{ll}
\Delta V_{\mathrm{BE}}=\left(\frac{k T}{q}\right) \ln \left(\frac{\text { highCD }}{\operatorname{lowCD}}\right) & \text { Sensitivity }(a)=138 \frac{\mu V}{{ }^{\circ} C} \\
\Delta V_{\mathrm{BE}}=a T & \text { for current density ratio of } 5
\end{array}
$$

\section{22 nm Sigma-Delta Based Thermal Sensor Architecture}

Better linearity is achieved using the Analog to Digital Converter (ADC) based thermal sensor [3,4]. In this architecture, the differential voltage is amplified and converted into a digital word by an ADC. A bandgap voltage reference circuit $(\mathrm{BG})$ can be used in order to supply a precision reference voltage for the ADC. This operating mode is called Vref mode. Another operating mode is creating the reference voltage internally in the TS. This is referred as Ratiometric mode (Figure 2). The ADC-based architecture has very good linearity over a wide range of temperatures. The accuracy of this architecture can be improved by using a calibration process during production. This architecture may produce accuracy of better than $\pm 0.5^{\circ} \mathrm{C}$ with old processes $[3,4,6,7]$.

The thermal sensor presented here was fabricated in the $22 \mathrm{~nm}$ process. The architecture includes an advanced sense stage combined with circuits that deal with process variations (chopping and dynamic element matching). A second order sigma-delta (SD) ADC is used both as an amplifier and as a converter that translates the temperature readings to a digital word. The SD converter includes correlated double sampling which improves its $1 / \mathrm{f}$ attenuation and offset performance and a custom made digital block. A synthesized block implements the sigma-delta decimation filters and provides a digital readout of the relative operating temperature as well as temperature alerts. This sigma-delta ADC based TS can be operated in two modes: Reference-based (Vref) and Ratiometric-based (Ratiometric). In the Ratiometric approach, the converter extracts bandgap voltages directly from the sense stage. Stabilization is achieved by feed-forward methodology. The sigma-delta modes are selectable through digital control manipulation only, without additional analog components. A thermal diode was also included within the TS for junction temperature sensing by a $\pm 1{ }^{\circ} \mathrm{C}$ sensor, as part of the calibration process. 
The TS has two main power modes: high sampling rate and power saving. In the power saving mode, the TS is switched on for a short time that enables reading of accurate temperature and most of the time the TS is off. In this method, the TS probes the temperature in frequencies of 10 to $100 \mathrm{samples} / \mathrm{s}$. In the high sampling rate mode the TS is continuously working with the sigma-delta modulator clocked at a lower frequency and samples the temperature in frequencies of $5 \mathrm{~K}$ to $45 \mathrm{~K}$ samples/s.

Figure 2. High level diagram of the Thermal Sensor.

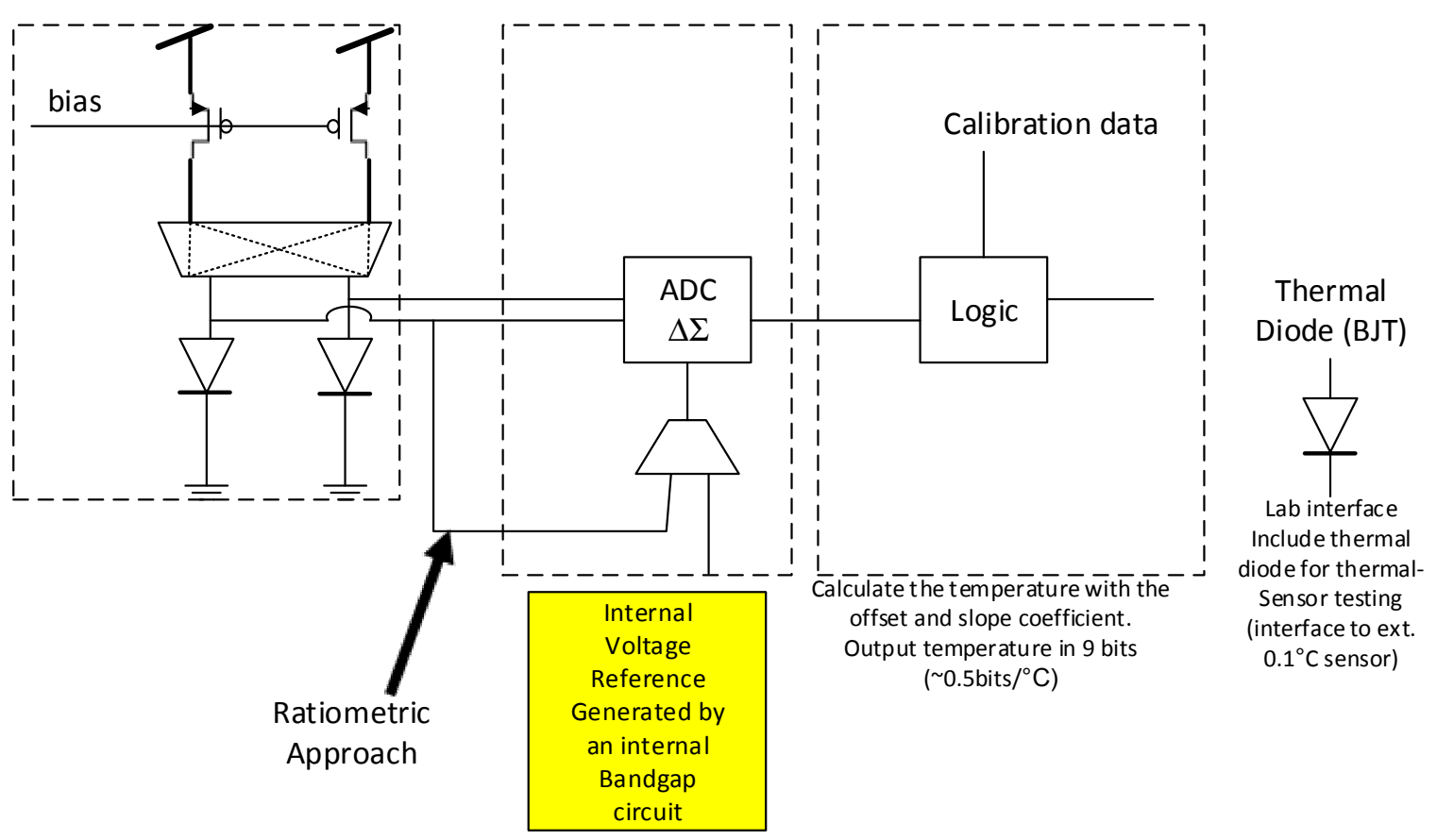

Device mismatches, such as between current mirrors and between diodes, are effectively treated by the advanced dynamic compensation circuit techniques. However, there are a few errors which require compensation during calibration if an improved accuracy is required. The sources of these errors are process variations of diode parameters, such as the ideality factor, variation of the conversion factor of the ADC dominated by capacitor mismatch, and Bandgap reference voltage variation in Vref mode only. The High Volume Manufacture (HVM) calibration result is stored in on-die fuses for use by the thermal sensor.

\section{The Sense Stage}

The sense stage diagram, presented in Figure 3, converts the junction temperature to a Proportional to Absolute Temperature (PTAT) voltage. It includes a matched pair of diodes and current mirrors with controlled current ratios (of 3.5, 4, 5, 6, 7, and 8). The absolute value of the mirrored current is controlled by an internal resistor and is equal to a reference voltage divided by the resistor value. The absolute current is designed to be a controlled value since it affects the current density within the diodes, thus affecting their performance as thermal sensing elements. In this work, the bias current of a single current source is nominally $15 \mu \mathrm{A}$. The current mirrors use gain boosting (regulated cascode) in order to increase current mirror output resistance, Rout, thus, further improves the current accuracy. These circuits paved the way to achieve accurate TS without the need of temperature calibration. 
Figure 3. Sense stage block diagram.

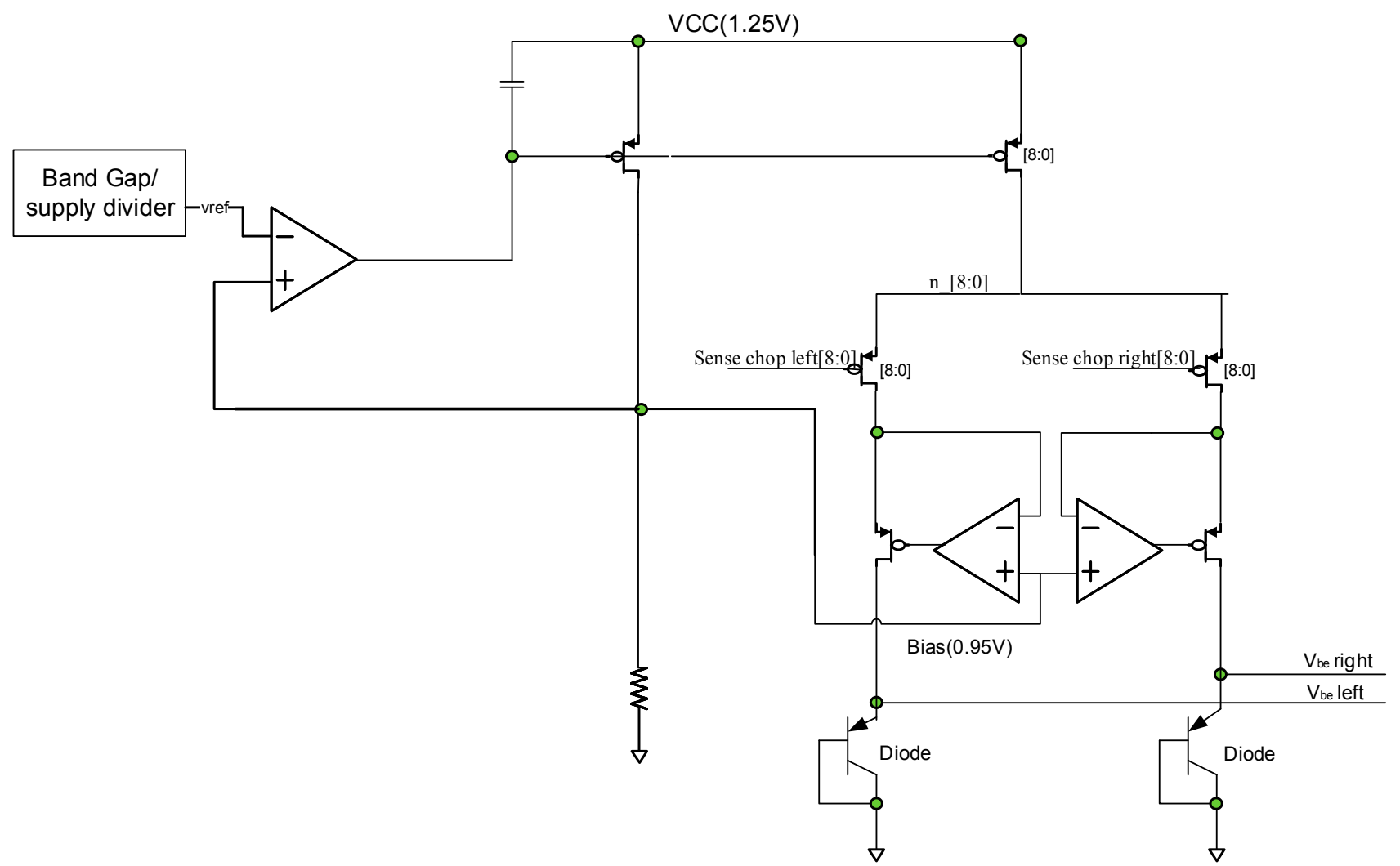

Current ratios are realized by utilizing nine identical current sources and by switching different subsets of the current to either the right or the left diode. In addition, current sources may be switched to a third diode, the waste diode (not shown in the figure), if they are not in use. The waste diode is implemented by driving current into dummies in the matched diode structure, thus avoiding the area penalty of extra circuitry and enable operating of the sense stage in higher frequency.

In order to obtain accurate current ratios resilient to mismatch, Dynamic Element Matching (DEM) is used. The DEM mechanism is realized by cyclically rotating the nine current source connections to the sense diodes and time-averaging the current value of the current mirrors.

Chopping is also used by the sense circuit in order to overcome diode mismatches. After selecting a current ratio, for example 7:2, it may be connected to the diodes in the reverse order as well (e.g., 2:7), thus changing the sign of $\Delta V_{\mathrm{BE}}$. This effectively modulates the signal on a carrier frequency, later to be demodulated by the sigma-delta converter. The chopping frequency is identical to the sampling frequency of the sigma-delta ADC so demodulation is back to DC.

From the circuit perspective, the sense stage is implemented with digital transistors on a relatively low power rail $(1.25 \mathrm{~V})$. The low threshold voltage of the digital transistors improves the circuits' headroom and improves the matching and Rout of the current mirrors and aids in achieving a good PSRR and an accurate TS in a small die area.

\section{The Sigma-Delta Modulator}

The sigma-delta modulator is a second order, one bit, switched-capacitor based design. Switched-capacitor circuits are frequently used for sigma-delta designs because they are fully 
compatible with digital CMOS processes. For low bandwidth applications, the over-sampling ratio (OSR) may be high enough so as not to limit the resolution of the ADC. A second order modulator is a preferred choice because it greatly reduces the stability problems of higher order modulators and decreases idle tone generation. It also lowers requirements for the OSR and thus for the gain of the integrator amplifiers. Integrator parameters (gains) are defined by the ratio of capacitors which is more accurate than absolute values of RC components in continuous time modulators. To reduce power supply noise and other distortions due to the common mode disturbance, a fully differential topology is chosen.

Correlated double-sampling (CDS) techniques and chopping are further used to decrease offset, 1/f noise and inaccuracy effects within the SD ADC.

The principles of the sigma-delta operation in the Vref-based and Ratiometric modes are presented in Figure 4. Charge balance is a fundamental property of any sigma-delta converter. This principle can be used to calculate the average signal transfer function of the modulator. The second stage of the SD-ADC affects mostly the quantization errors and thus does not change the essential transfer function of the ADC.

Figure 4. Sigma-delta Analog to Digital Converter (ADC) operation principle-(A) Vref mode; and (B) ratiometric mode.

(A)

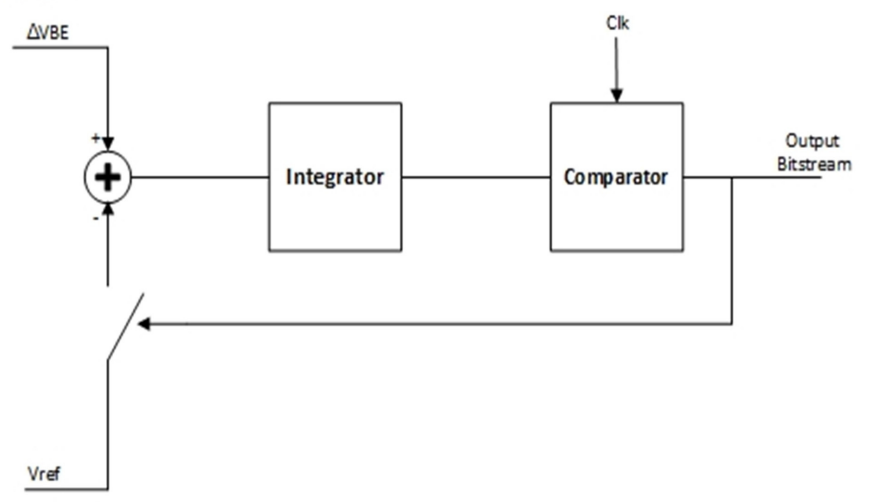

(B)

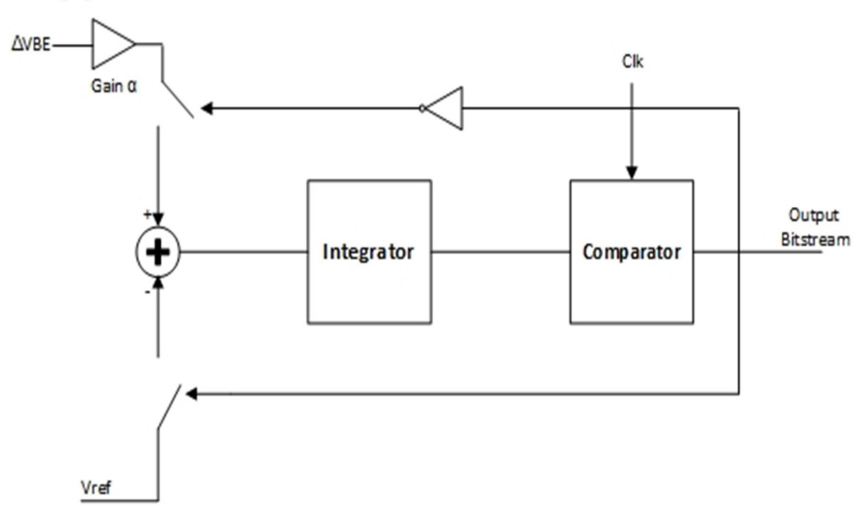

Charge balance principle:

$$
\begin{aligned}
& -T_{\text {off }} \times \Delta V_{\mathrm{BE}}=T_{\text {on }} \times\left(\Delta V_{\mathrm{BE}}-V_{\mathrm{REF}}\right) \\
& \Rightarrow \\
& \frac{T_{\text {off }}}{T_{\text {on }}}=\frac{V_{\mathrm{REF}}}{\Delta V_{\mathrm{BE}}}-1 \Rightarrow \\
& \left(1+\frac{T_{\text {off }}}{T_{\text {on }}}\right)^{-1}=\frac{T_{\text {on }}}{T_{\text {on }}+T_{\text {off }}}=\frac{T_{\text {on }}}{T}=\frac{\Delta V_{\mathrm{BE}}}{V_{\mathrm{REF}}}
\end{aligned}
$$

Charge balance principle:

$$
\begin{aligned}
& T_{\text {off }} \times \alpha \times \Delta V_{\mathrm{BE}}=-T_{\text {on }} \times\left(-V_{\mathrm{BE}}\right) \\
& \Rightarrow \\
& \frac{T_{\text {off }}}{T_{\text {on }}}=\frac{V_{B E}}{\alpha \Delta V_{\mathrm{BE}}} \Rightarrow \\
& \left(1+\frac{T_{\text {off }}}{T_{\text {on }}}\right)^{-1}=\frac{T_{\text {on }}}{T_{\text {on }}+T_{\text {off }}}=\frac{\alpha \Delta V_{\mathrm{BE}}}{V_{\mathrm{BE}}+\alpha \Delta V_{\mathrm{BE}}}
\end{aligned}
$$

Charge-balance mathematics are applied for demonstration to the reference-based sigma-delta, showing how the voltage ratio $\left(\Delta V_{\mathrm{BE}} / V_{\mathrm{REF}}\right)$ is converted into an average duty ratio (or " 1 " density ratio) by the converter.

Applying the same principle to the ratiometric construction shows how the " 1 " density ratio in the bitstream of the Ratiometric modulator is caused by the ratio: 


$$
\frac{\alpha \Delta V_{\mathrm{BE}}}{V_{\mathrm{BE}}+\alpha \Delta V_{\mathrm{BE}}}
$$

where $\alpha$ is the preamp gain and $\Delta V_{\mathrm{BE}}$ and $V_{\mathrm{BE}}$ are the diodes' voltages generated by the sense stage. Proper selection of $\alpha$ will cause the denominator to balance the complementary to absolute temperature (CTAT) behavior of $V_{\mathrm{BE}}$ against the proportional to absolute temperature (PTAT) behavior of $\Delta V_{\mathrm{BE}}$, achieving the reference Vref within the sigma-delta converter itself. This phenomena is named Ratiometric sigma-delta [6,7], and is used in the thermal sensor in order to omit the bandgap from the sense path (utilizing instead the natural "bandgap" that is already embedded in the sense stage).

The architecture of the sigma-delta converter is presented in Figure 5. The architecture is based on the converter presented by Pertijs and al [7]. Each amplifier constitutes an integrator, thus this circuit is a second order design.

The first integrator incorporates an offset cancelling scheme. It stores the offset on the input capacitors with reverse polarity and during the integration phase the stored offset is subtracted from the integrated signal. The input capacitors to the first amplifier are used to implement a controlled gain. If FS3 is off, then the bucket capacitor of the integrator consists of Cs1 only. Therefore the gain of the integrator would be Cs1/Cint1. However, if Cs2 is connected, the gain would be (Cs1 + Cs2)/Cint1. We use this feature in Vref-mode to boost the $\Delta V_{\mathrm{BE}}$ signal by a factor of eight, and in the Ratiometric mode as part of the gain $\alpha$. This eliminates the need for a preamplifier between the sense stage and the ADC converter, thus saving power and area. Additional gain may be obtained, if required, by performing several cycles of integration before the comparator clock $\left(\Phi_{\mathrm{cmp}}\right)$ is asserted.

Figure 5. Second order sigma-delta converter.

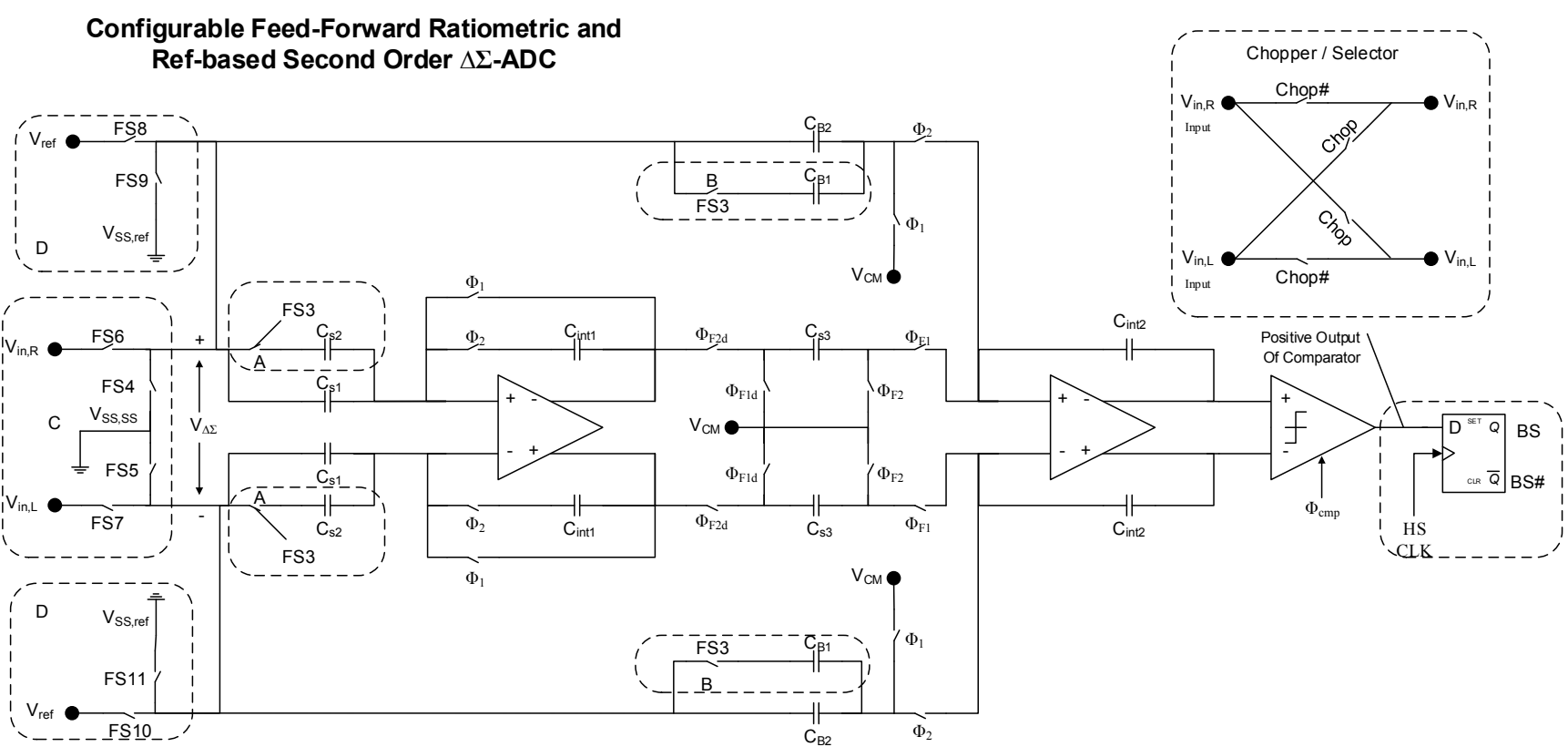

The second stage is a switch capacitor integrator. Feed-forward path stabilization causes low swing at the opamps' outputs so less distortion is expected. No DC component above CM voltage exists at the first integrator output. However, the feed-forward method makes the transfer function depend on capacitors' mismatch. 
The ADC is controlled by several clocks ( $\Phi 1, \Phi 1 \mathrm{t}$ and $\Phi 2, \Phi 2 \mathrm{t}$ and their delayed versions) and functional switch controls: FS3-FS11. This set of control signals is generated by a custom logic circuit within the ADC, Which selects values for the functional switches in two different modes of operation: Vref-based and Ratiometric-based converters.

The bit stream output feeds a three-stage (CIC) filter that removes high-frequency noise and frames the sampled signal into nine bit samples.

\section{The TS Top Hierarchy and Layout}

As mentioned above, this TS IP is designed for SoC projects and therefore it is designed as a self-contained collateral that includes all the infrastructure for supporting the requirements of multiple projects. In addition to the TS analog core circuit of $0.013 \mathrm{~mm}^{2}$, there are other pieces of collateral, including: (1) a thermal diode used in the calibration process; (2) a logic core required for digital DSP, filtering and processing the SD-ADC output; (3) two analog ports for Si testing that are connected directly to critical points in the circuit or through analog to frequency converters (A2F); (4) clamps and decoupling capacitors; and (5) Jtag interface for independent accessibility. The thermal sensor layout picture and specifications are presented in Figure 6.

Figure 6. The self-contained thermal sensor layout picture including above the TS core area also thermal diode, clamp, supply decoupling and logic.

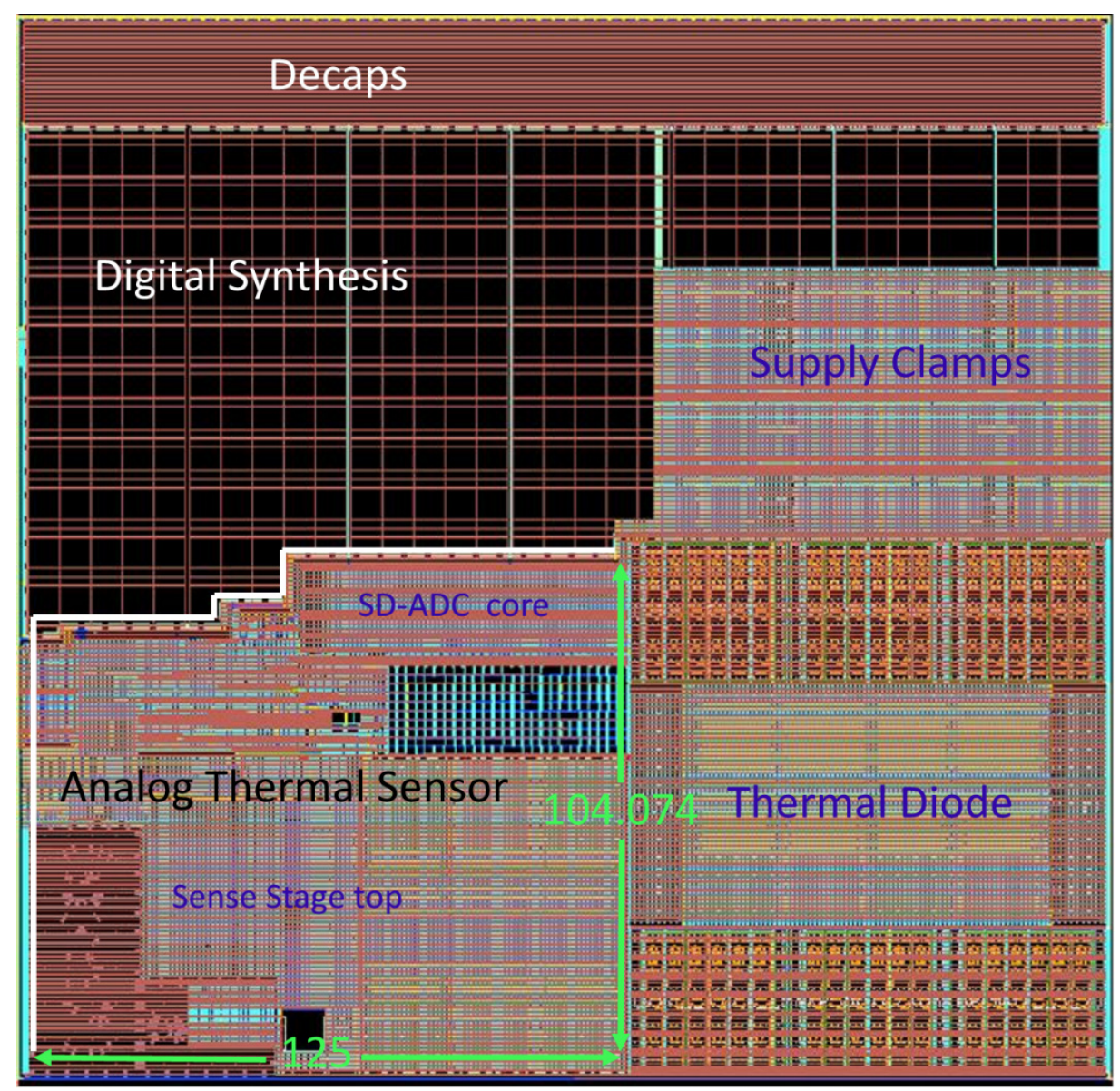


The analog core works on a $1.25 \mathrm{~V}$ although the digital transistors do not support such direct voltage stress. In order to overcome the over stress limitations of the transistors, advanced techniques were used, such as adding protecting devices and start-up circuits. The use of digital transistor with higher supply voltages enables high bandwidth, relatively high sampling rates, enough headroom range and very small die area. The ADC improved performance together with a configurable and fast digital filter, reduces the latency time of temperature readout, and enables the TS to work with a wide envelope of sample frequencies with optimized power options.

\section{Results}

The suggested SD-ADC based thermal sensor was implemented in a $22 \mathrm{~nm}$ process and was verified in two generations of test chips on typical and skew material. The evaluation boards included an adapter for a thermal head, which forces a specific temperature and an external component for checking the internal thermal diode for measuring the junctional temperature. Extracting the temperature inaccuracy of each chip was done in Ratiometric mode at a temperature range of $0-110{ }^{\circ} \mathrm{C}$.

Figure 7A depicts the temperature reading errors of typical dies after one temperature calibration at $100{ }^{\circ} \mathrm{C}$ in Ratiometric mode with supply voltage of $1.25 \mathrm{~V}$. The error for a wide range of temperature is smaller than $\pm 1{ }^{\circ} \mathrm{C}$. The results of the same chips only untrimmed (non-calibrated) demonstrates an inaccuracy of smaller than $\pm 1{ }^{\circ} \mathrm{C}$ (Figure 7B). Note that these results include the error induced by the measuring procedure done by the thermal diode.

Figure 7. Temperature error versus junction temperature of different typical chips: (A) calibrated in one temperature and; (B) untrimmed.

(A)

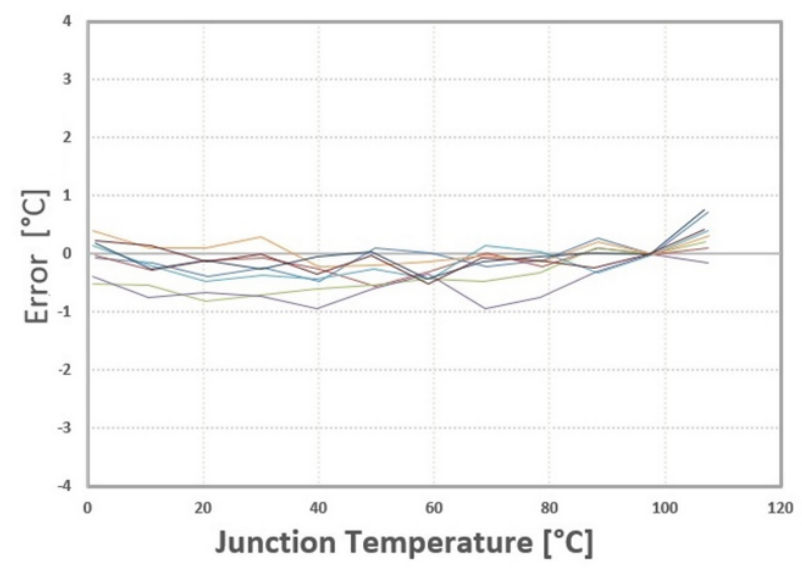

(B)

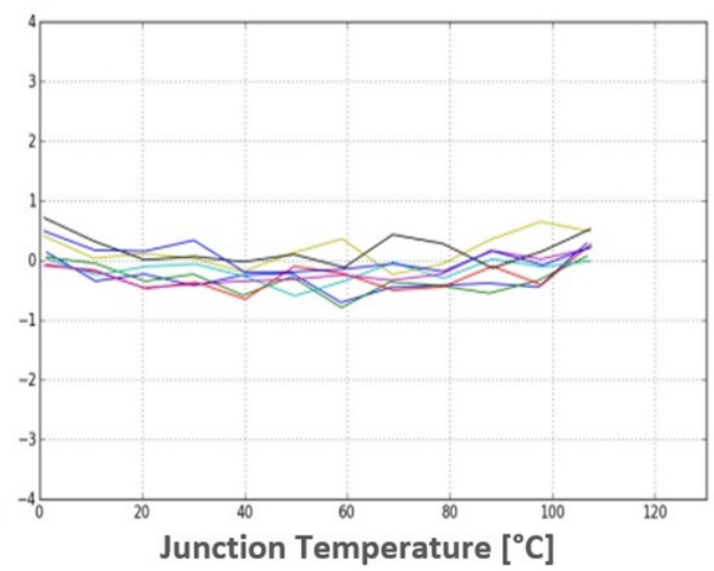

The TS circuit is differential and is very insensitive to power supply variation. The temperature error curves with various supply voltages of $1.25 \mathrm{~V} \pm 5 \%$, and $1.35 \mathrm{~V} \pm 5 \%$ are presented in Figure 8 with an inaccuracy of smaller than $\pm 1.8{ }^{\circ} \mathrm{C}$ demonstrating a good immunity to supply voltage. In addition, simulations support these results and demonstrate a high PSRR of $>50 \mathrm{~dB}$ for the $\Delta V_{\mathrm{BE}}$ signal over a wide frequency range (not presented). The conclusion is that a voltage regulator is not required for this TS supply unlike other TS in the industry that require a voltage regulator [5]. 
Figure 8. Temperature error versus junction temperature of 16 chips in Ratiometric mode with various supply voltages of (A) $1.25 \mathrm{~V} \pm 5 \%$ and (B) $1.35 \mathrm{~V} \pm 5 \%$.
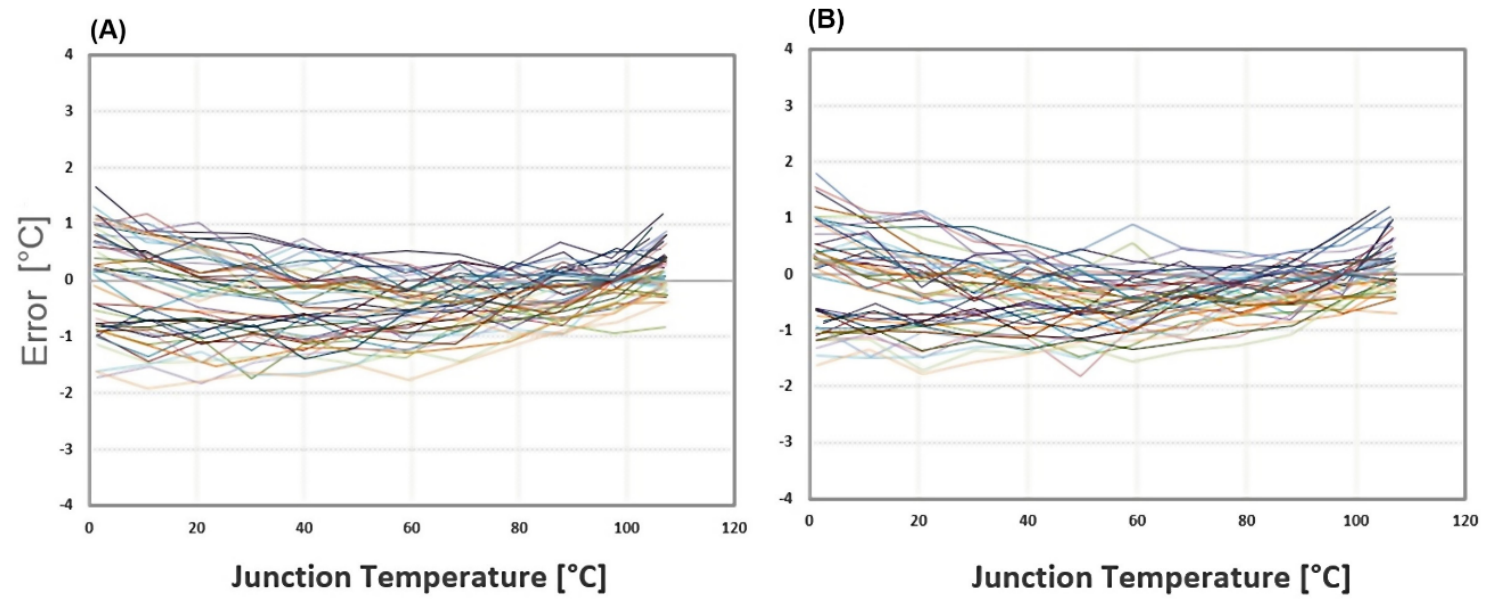

The TS power consumption from the analog supply voltage $(1.25 \mathrm{~V})$ appears in Table 1 . In the power saving mode in which the sampling rate is low (10-100 Sps), the power consumption is significantly reduced from $\sim 1 \mathrm{~mW}$ in high sampling rate mode down to $18 \mu \mathrm{W}$. In order to support frequently disabling/enabling the SD ADC, we increased the clock frequency of the sigma-delta modulator. We also reduced the delay on the digital filters by using only three stages of (CIC) filter. A quite short latency for the SD ADC and digital filter was achieved of approximately $40 \mu$ s, while maintaining the ADC SNR. The power numbers for both high sampling rate mode (done for the max $45 \mathrm{KSps}$ ) and power saving mode are calculated over 16 different chips. The "typical" results represent the average results obtained from typical die, while the "fast" results represent the absolute highest consumption measured for "fast" skew SI.

Table 1. Thermal Sensor power consumption for different sampling rates. "Typical" are the average numbers for typical die where "fast" results represent the absolute highest consumption measured for "fast" skew SI.

\begin{tabular}{cccccc}
\hline Power mode & Corner/Skew & $\begin{array}{c}\text { Temp } \\
\left(\mathbf{C}^{\circ}\right)\end{array}$ & $\begin{array}{c}\text { Analog } \\
\text { supply (V) }\end{array}$ & $\begin{array}{c}\text { Analog current } \\
\text { consumption }(\boldsymbol{\mu A})\end{array}$ & $\begin{array}{c}\text { Analog power } \\
\text { consumption }(\boldsymbol{\mu W})\end{array}$ \\
\hline $\begin{array}{c}\text { Power saving mode } \\
(10 \text { Sps) }\end{array}$ & Typical & 60 & 1.25 & 14 & 18 \\
$\begin{array}{c}\text { Power saving mode } \\
(10 \text { Sps) }\end{array}$ & Fast & 110 & 1.31 & 15 & 20 \\
$\begin{array}{c}\text { Power saving mode } \\
(100 \text { Sps })\end{array}$ & Typical & 60 & 1.25 & 52 & 65 \\
$\begin{array}{c}\text { Power saving mode } \\
(100 \text { Sps })\end{array}$ & Fast & 110 & 1.31 & 60 & 79 \\
$\begin{array}{c}\text { High sampling rate } \\
\text { High sampling rate }\end{array}$ & Typical & 60 & 1.25 & 780 & 975 \\
\hline
\end{tabular}

Finally, the untrimmed inaccuracy, without using any calibration, is quite low (Figure 9) paving the way for using this TS without calibration for most SoC products. The error for 29 devices is $\pm 2{ }^{\circ} \mathrm{C}$ in Ratiometric mode (Figure 9A). The average temperature error is $<0.5^{\circ} \mathrm{C}$ and the resolution is 
$\pm 0.25{ }^{\circ} \mathrm{C}$ without any averaging of reading samples. The one sigma error is less than $1{ }^{\circ} \mathrm{C}$ (Figure 9B) and the three sigma error is less than $\pm 3{ }^{\circ} \mathrm{C}$. Such inaccuracy is close to the results achieved by a practical calibration process such as using thermal diode. It means that calibration is unnecessary.

Figure 9. Untrimmed inaccuracy of thermal sensors in Ratiometric mode. (A) Temperature error versus junction temperature without calibration for 29 devices; (B) Statistics of errors presented in A. The yellow rectangle indicates the average error in a specific temperature and the error bar indicates the standard deviation $(1 \sigma)$ of the error.

(A)

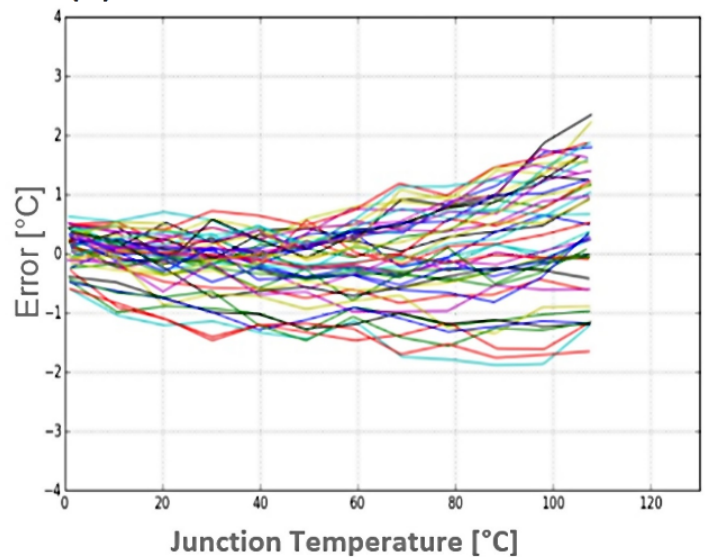

(B)

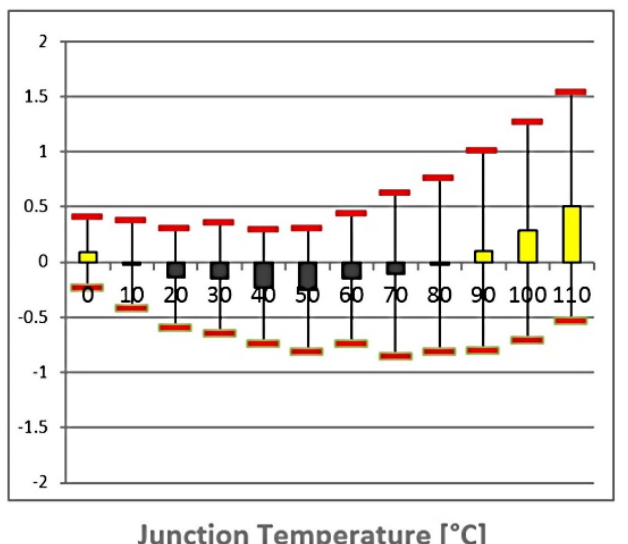

In Table 2 you can see a comparison made with other TS available in the literature. The TS described in this article shows a very good performance in power, area and speed while maintaining good accuracy even without calibration.

Table 2. Comparison with other TSs in the literature. Abs-absolute temperature error.

\begin{tabular}{|c|c|c|c|c|c|c|}
\hline Reference & $\begin{array}{c}\text { Sebastiano } \\
{[3]}\end{array}$ & Souri [4] & $\begin{array}{c}\text { Lakdawala } \\
{[\text { [5] }}\end{array}$ & Shor [2] & $\begin{array}{c}\text { "High } \\
\text { Sampling } \\
\text { Rate" } \\
\end{array}$ & $\begin{array}{c}\text { "Power } \\
\text { Saving Mode" }\end{array}$ \\
\hline Source/Year & JSSC 2010 & JSSC 2013 & JSSC 2009 & JSSC 2013 & This work & This work \\
\hline Technology & $65 \mathrm{~nm}$ & $0.16 \mu \mathrm{m}$ & $32 \mathrm{~nm}$ & $22 \mathrm{~nm}$ & $22 \mathrm{~nm}$ & $22 \mathrm{~nm}$ \\
\hline Area $\left(\mathrm{mm}^{2}\right)$ & 0.1 & 0.08 & 0.02 & 0.0061 & 0.013 & 0.013 \\
\hline Voltage Regulator & not required & not required & required & not required & not required & not required \\
\hline Calibration & not required & not required & not required & required & not required & not required \\
\hline Supply Voltage (V) & $1.2-1.3$ & $1.5-2$ & 1.05 & 1.35 & 1.25 & 1.25 \\
\hline Current & $8.3 \mu \mathrm{A}$ & $3.4 \mu \mathrm{A}$ & $1.5 \mathrm{~mA}$ & $1.03 \mathrm{~mA}$ & $780 \mu \mathrm{A}$ & $15-60 \mu \mathrm{A}$ \\
\hline Speed (samples/s) & 2.2 & $10-188$ & $1 \mathrm{~K}$ & $2 \mathrm{~K}-20 \mathrm{~K}$ & $5 \mathrm{~K}-45 \mathrm{~K}$ & $10-100$ \\
\hline Energy/Sample (J) & $4.5 \mu$ & $27 n-440 n$ & $1.6 \mu$ & $69.5 n-0.69 \mu$ & $30 n-270 n$ & $750 \mathrm{n}-1.88 \mu$ \\
\hline $\begin{array}{c}\text { Accuracy with/out } \\
\text { calibration }\left({ }^{\circ} \mathrm{C}\right)\end{array}$ & $0.2 / 0.5(3 \Sigma)$ & $\begin{array}{c}0.15[0.25] / \\
0.6[0.8](3 \Sigma)\end{array}$ & /5 (abs) & 1.5 (abs) & $1.8 / 2(\mathrm{abs})$ & $1.8 / 2$ (abs) \\
\hline Range & -70 to 125 & -55 to 125 & -10 to 110 & -10 to 100 & -10 to 110 & -10 to 110 \\
\hline $\begin{array}{c}\text { Accuracy FOM } \\
\left.\left(\mathrm{J}^{2}\right)^{2}\right)\end{array}$ & $47.3 \mathrm{n}$ & $0.75 \mathrm{n}$ & $27.7 \mu$ & $679 n$ & $67.5 \mathrm{n}$ & $1.6 \mu$ \\
\hline
\end{tabular}

* Where it was possible the values were taken from the article. The rest calculated by using the equation at [4]. 


\section{Summary}

In this paper we presented a precise low power integrated thermal sensor. The architecture uses advanced features such as dynamic element matching, precise regulated cascode current mirror, chopping, and correlated double sampling to overcome accuracy and matching issues. Silicon validation performed demonstrates immunity to process variation and an error $< \pm 1.8^{\circ} \mathrm{C}$ for a range of $0-110{ }^{\circ} \mathrm{C}$ with a single temperature calibration.

Some of the configurability options were demonstrated, showing reference-based and Ratiometric operation. In the Ratiometric mode, a reference may not be needed as it is extracted by the ADC from the sense stage itself. Many additional configuration options, such as remote diode interfaces, signal path gain control, diode current ratio control and digital post processing options make this architecture a fertile testing ground for the exploration of temperature sensing techniques.

The untrimmed inaccuracy of the thermal sensor is less than $\pm 3{ }^{\circ} \mathrm{C}(3 \sigma)$ across the entire range. Reducing the calibration process will significantly reduce the tester cost for SoC products.

Over all, the silicon results show a unique ability to trade between high sampling rates and low power consumption up to $18 \mu \mathrm{W}$ while retaining high accuracy without calibration in a small die area.

\section{Acknowledgments}

The authors would like to acknowledge the following team members from Intel: Vitali Rahinski, Yosi Sanhedrai, Elad Kuperberg, Tomer Fael, Guillermo Libashevsky, Gilad Peleg and Michael L Tollen who contributed a lot for the design and layout of the thermal sensor and also to Emanuel Natanov and Guy Avigad for leading the post-Si validation.

\section{Author Contributions}

Roee Eitan was responsible for the design and testing of the circuits described here. Ariel Cohen guided this research, reviewed the proposed circuits and edited this paper.

\section{Conflicts of Interest}

The authors declare no conflict of interest.

\section{References}

1. JEDEC Standard. FBDIMM: Advanced Memory Buffer (AMB); JESD82-20; JEDEC Solid State Technology Association: Arlington, VA, USA, 2007.

2. Shor, J.S.; Luria, K. Miniaturized BJT-Based Thermal Sensor for Microprocessors in 32- and 22-nm Technologies. IEEE J. Solid-State Circuits 2013, 48, 2860-2867.

3. Sebastiano, F.; Breems, L.; Makinwa, K.A.A.; Drago, S.; Leenaerts, D.M.W.; Nauta, B. A 1.2-V $10-\mu \mathrm{W}$ NPN-based temperature sensor in 65-nm CMOS with an inaccuracy of $0.2{ }^{\circ} \mathrm{C}(3 \sigma)$ from $70{ }^{\circ} \mathrm{C}$ to $125^{\circ} \mathrm{C}$. IEEE J. Solid-State Circuits 2010, 45, 312-313. 
4. Souri, K.; Chae, Y.; Makinwa, K.A.A. A CMOS temperature sensor with a voltage-calibrated inaccuracy of $\pm 0.15{ }^{\circ} \mathrm{C}(3 \sigma)$ from $55{ }^{\circ} \mathrm{C}$ to $125{ }^{\circ} \mathrm{C}$. IEEE J. Solid-State Circuits 2013, 48, 292-301.

5. Lakdawala, H.; Li, Y.W.; Raychowdhury, A.; Taylor, G.; Soumyanath, K. A 1.05 V $1.6 \mathrm{~mW}$ $0.45{ }^{\circ} \mathrm{C} 3 \sigma$-resolution $\Delta \Sigma$-based temperature sensor with parasitic-resistance compensation in 32 nm CMOS. IEEE J. Solid-State Circuits 2009, 44, 340-341.

6. Pertijs, M.A.; Makinwa, K.A.A.; Huijsing, J. A CMOS smart temperature sensor with a 3 sigma inaccuracy of $0.1 \mathrm{C}$ from $55 \mathrm{C}$ to $125 \mathrm{C}$. IEEE J. Solid-State Circuits 2005, 40, 2805-2815.

7. Pertijs, M.A.; Niederkorn, A.; Ma, X.; McKillop, B.; Bakker, A.; Huijsing, J.H. A CMOS smart temperature sensor with a 3 sigma inaccuracy of $0.5{ }^{\circ} \mathrm{C}$ from $50{ }^{\circ} \mathrm{C}$ to $120{ }^{\circ} \mathrm{C}$. IEEE J. Solid-State Circuits 2005, 40, 454-461.

8. Wang, G.; Meijer, G.C.M. The temperature characteristics of bipolar transistors fabricated in CMOS technology. Sens. Actuators A 2000, 87, 81-89.

9. Duarte, D.E.; Geannopoulos, G.; Mughal, U.; Wong, K.L.; Taylor, G. Temperature Sensor Design in a High Volume Manufacturing $65 \mathrm{~nm}$ CMOS Digital Process. In Proceedings of the IEEE 2007 Custom Integrated Circuits Conference, San Jose, CA, USA, 16-19 September 2007.

(C) 2014 by the authors; licensee MDPI, Basel, Switzerland. This article is an open access article distributed under the terms and conditions of the Creative Commons Attribution license (http://creativecommons.org/licenses/by/4.0/). 